\title{
Carotid DSA based CFD simulation in assessing the patient with asymptomatic carotid stenosis: a preliminary study
}

\author{
Dong Zhang ${ }^{1 \dagger}$, Pengcheng $\mathrm{Xu}^{2 \dagger}$, Hongyu Qiao ${ }^{1}$, Xin Liư ${ }^{2}$, Liangping Luo ${ }^{1}$, Wenhua Huang ${ }^{2}$, Heye Zhang ${ }^{3^{*}}$ \\ and Changzheng Shi ${ }^{*}$ (D)
}

\author{
*Correspondence: \\ hy.zhang@siat.ac.cn; \\ tsczcn@jnu.edu.cn \\ ${ }^{\dagger}$ Dong Zhang and \\ Pengcheng Xu contributed \\ equally to this work \\ ${ }^{1}$ Department of Medical \\ Imaging Center, The First \\ Affiliated Hospital, Jinan \\ University, No. 613, Huangpu \\ Road West, Tianhe District, \\ Guangzhou 510630, \\ Guangdong Province, China \\ ${ }^{3}$ Institute of Advanced \\ Computing and Digital \\ Engineering, Shenzhen \\ Institutes of Advanced \\ Technology, 1068 Xueyuan \\ Ave, Xili University Town, \\ Nanshan, Shenzhen 518055, \\ Guangdong Province, China \\ Full list of author information \\ is available at the end of the \\ article
}

\begin{abstract}
Background: Cerebrovascular events are frequently associated with hemodynamic disturbance caused by internal carotid artery (ICA) stenosis. It is challenging to determine the ischemia-related carotid stenosis during the intervention only using digital subtracted angiography (DSA). Inspired by the performance of well-established FFRct technique in hemodynamic assessment of significant coronary stenosis, we introduced a pressure-based carotid arterial functional assessment (CAFA) index generated from computational fluid dynamic (CFD) simulation in DSA data, and investigated its feasibility in the assessment of hemodynamic disturbance preliminarily using pressure-wired measurement and arterial spin labeling (ASL) MRI as references.
\end{abstract}

Methods: The cerebral multi-delay multi-parametric ASL-MRI and carotid DSA including trans-stenotic pressure-wired measurement were implemented on a 65-year-old man with asymptomatic unilateral (left) ICA stenosis. A CFD simulation using simplified boundary condition was performed in DSA data to calculate the CAFA index. The cerebral blood flow (CBF) and arterial transit time (ATT) of ICA territories were acquired.

Results: CFD simulation showed good correlation $(r=0.839, P=0.001)$ with slight systematic overestimation (mean difference -0.007 , standard deviation 0.017 ) compared with pressure-wired measurement. No significant difference was observed between them $(P=0.09)$. Though the narrowing degree of in the involved ICA was about $70 \%$, the simulated and measured CAFA (0.942/0.937) revealed a functionally nonsignificant stenosis which was also verified by a compensatory final CBF (fronto-temporal/frontoparietal region: $51.58 / 45.62 \mathrm{ml} / 100 \mathrm{~g} / \mathrm{min}$ ) and slightly prolonged ATT (1.23/1.4 s) in the involved territories, together with a normal left-right percentage difference $(2.1-8.85 \%)$.

Conclusions: The DSA based CFD simulation showed good consistence with invasive approach and could be used as a cost-saving and efficient way to study the relationship between hemodynamic disorder caused by ICA stenosis and subsequent perfusion variations in brain. Further research should focus on the role of noninvasive pressure-based CAFA in screening asymptomatic ischemia-causing carotid stenosis.

Keywords: CFD, Carotid stenosis, Perfusion, Angiography 


\section{Background}

Internal carotid stenosis is the main cause of hemodynamic disturbance and the subsequent stroke in brain [1]. Furthermore, asymptomatic internal carotid stenosis affects approximately $7 \%$ of women and more than $12 \%$ men older than 70 years, and most of them have the stenosis $\geq 50 \%$ [2]. However, most of the patients are not aware of this condition until fatal events happen. The management of patients with asymptomatic internal carotid stenosis is challenging in clinical practice because the definition of the high-risk patients with severe asymptomatic carotid stenosis remains unclear [3-5].

At present, angiography is the clinical standard to evaluate the severity of carotid stenosis, including Doppler ultrasonography, computed tomography angiography (CTA), magnetic resonance angiography (MRA), and digital subtraction angiography (DSA) [6-8]. However, these routine methods can only detect the anatomical carotid stenosis. According to Kasner's research, the probability of stroke is 0.08 in 1 year to the patients with stenosis $50-69 \%$, and is 0.23 to the patients with stenosis $\geq 70 \%$ [6]. The alteration of hemodynamic function plays an important role in assessing the risk of stroke to patients with asymptomatic internal carotid stenosis $[9,10]$.

The fraction flow reserve (FFR) has been well-established in evaluating the hemodynamic characteristics of coronary artery stenosis [11]. Using a pressure-sensing guidewire, Han and Liu have validated the feasibility of pressure gradient measurement in intracranial large arteries and vertebral artery in clinic, respectively [12, 13]. However, guidelines for the application of pressure wire in carotid arteries has not yet been established, and the high cost and invasive practice may be the main reasons [14, 15]. The advance of computational fluid dynamic (CFD) could be an alternative and efficient tool to make up for its shortages, and the noninvasive FFR measurement based on coronary CTA data have presented good performance in diagnosing the ischemic lesions [16, 17]. Furthermore, hemodynamic characteristics and flow patterns on the site of carotid stenosis can also be calculated and visualized by combining the contrast-enhancement MR angiography (CE-MRA) or DSA with CFD [18-20]. But few focused on the trans-stenotic pressure gradient measurement of internal carotid artery, and its relationship with risk of ischemic stroke in brain still lacks enough evidence [21].

Perfusion is an important parameter to estimate dysfunction in the level of brain tissues [22, 23]. The compromised cerebral blood flow (CBF) caused by internal carotid stenosis indicates a risk for future ischemic stroke in brain $[9,24]$. Arterial spin labeling (ASL) has emerged as a useful tool for CBF evaluation in clinical practice because of its noninvasive, nonradioactive and nonpoisonous advantages [25-27]. Furthermore, ASL is highly susceptible to the arterial transit time (ATT) which is associated with collateral blood flow [28], and it would be ideal to apply ASL with multiple post-labeling delay (PLD) time to estimate the CBF and ATT simultaneously [29].

In this study, based on a case with asymptomatic unilateral internal carotid stenosis, we introduced a pressure-based carotid arterial functional assessment (CAFA) index generated from CFD simulation in DSA data, and further investigated its feasibility in the assessment of hemodynamic disturbance preliminarily using invasive pressure-wired measurement and multi-delay multi-parametric ASL-MRI as references. 


\section{Methods}

This study was conducted in accordance with the principles of the Declaration of Helsinki and met the requirement of medical ethics. The Local Ethical Review Committee approved this research. Since this study was retrospective in nature, the informed consent was waived and anonymized data was used for analysis.

\section{Case presentation}

A 65-year-old man with a history of hypertension for 11 years and type 2 diabetes mellitus (T2DM) for 3 years treated with amlodipine and perindopril once a day and insulin injection every day. His hypertension and T2DM were considered to be controlled. $\mathrm{He}$ was recommended a Doppler ultrasonography of cervical arteries as a screening due to his vascular risk factors. Doppler ultrasonography showed an atherosclerotic stenosis at the proximal site of left internal carotid artery (ICA) which was characterized as homogeneous plaque. There was a peak systolic velocity increase of $168 \mathrm{~cm} / \mathrm{s}$ at the site of stenosis. The morphology data presented a carotid stenosis of $69 \%$. And then, DSA were performed and the results confirmed a severe stenosis at the proximal site of left ICA, and the degree of narrowing was also estimated about $70 \%$ according to the NASCET criteria (North American Symptomatic Carotid Endarterectomy Trial). A pressure-sensing guidewire was used when the invasive carotid angiography was performed. Besides, brain MRI revealed no abnormal lesions. For further evaluation, a pseudo-continuous arterial spin labeling (pCASL) MRI data with multiple post-labeling delay (PLD) was acquired in this case.

\section{MRI protocols and data processing}

MRI examination was performed on a 3.0 T MR system (GE Healthcare Discovery MR 750 , Waukesha, WI) with 8 channel head coils. The pCASL scans were performed using background suppressed and a stack-of-spirals 3D-fast-spin echo imaging sequence with the following parameters: PLD $=1.0 / 2.0 / 3.0 \mathrm{~s}$; TR/TE, 4658/11.1 ms; FOV, $240 \times 240 \mathrm{~mm}$; matrix, $512 \times 8$; section thickness, $3.0 \mathrm{~mm}$; bandwidth $62.5 \mathrm{kHz}$ and NEX, 3.

CBF maps at each single PLD were obtained offline using an independent workstation (Advantage Workstation 4.5, GE Healthcare) based on the following equations:

$$
\mathrm{WD}=\left[\sum_{i=1}^{4} w(i) \Delta M(i)\right] /\left[\sum_{i=1}^{4} \Delta M(i)\right]
$$

where $\Delta M(i)$ is the mean perfusion difference images for each PLD, and $w(i)$ is the PLD (1.0/2.0/3.0 s). A weighted delay WD is calculated by Eq. (1) and converted into ATT or $\delta$ based on the theoretical relationship between WD and ATT [27]. CBF at each delay,f(i), is calculated using the measured ATT map and the Eq. (2).

$$
f(i)=\frac{\lambda \Delta M i R_{1 a}}{2 \alpha M_{0}\left[\exp \left((\min (\delta-w(i), 0)-\delta) R_{1 a}\right)-\exp \left(-(\tau+w(i)) R_{1 a}\right)\right]}
$$

where $R_{1 a}(=0.72 / 0.61 / \mathrm{s}$ at $1.5 / 3 \mathrm{~T})$ is the longitudinal relaxation rate of blood, $M_{0}$ is the equilibrium magnetization of brain tissue, $\alpha(=0.8)$ is the tagging efficiency, $\tau(=1.5 \mathrm{~s})$ is 
the duration of labeling pulse, $w(i)$ is the PLD (1.0/2.0/3.0 s), $\lambda(=0.9 \mathrm{~g} / \mathrm{ml})$ is the blood/ tissue water partition coefficient. The final CBF is defined as the mean of estimated CBF at each PLD [30].

\section{Digital subtraction angiography and pressure-wired measurement}

The 3D-DSA examination of cerebral and carotid arteries was carried out with a rotational angiographic system (Artis zeego, Siemens Healthcare, Forchheim, Germany) and was done with nonionic contrast medium (Visipaque, GE Healthcare) $2-2.4 \mathrm{ml} / \mathrm{s}$ with a total injection volume of $15-18 \mathrm{ml}$. The 3D-DSA was performed with a C-arm rotation of $180^{\circ}$.

According to the protocol introduced by Han [12], the reference blood pressure ( $\mathrm{mPa})$ was acquired at the cervical portion of internal carotid artery $(\mathrm{C} 1)$ by the catheter, and then a pressure wire (PressureWire Aeris/Certus, St. Jude Medical, St. Paul, USA) was positioned distal to the stenosis of interest (at least $3 \mathrm{~cm}$ downstream of the lesion) and the mean distal arterial pressure $(\mathrm{mPd})$ was measured. Finally, the guidewire was pulled back, and the pressure gradient was record continuously. The invasive CAFA was calculated by dividing the $\mathrm{mPd}$ by $\mathrm{mPa}$.

\section{CFD configuration}

\section{Boundary condition}

The volumetric flow rate was derived using the lumen volume divided by the transport time. One can easily confirm two frames when the blood flew through the inlet of the reconstructed vessels. Based on DSA data set, we could obtain the flux as well as the lumen volume during two frames. The volumetric flow rate divided by area of the inlet was defined as the inlet boundary. As for the outlets, outflow conditions were applied.

The blood motion was governed by Navier-Stokes equations as

$$
\begin{aligned}
& \rho\left(\frac{\mathrm{du}}{\mathrm{dt}}+\mathrm{u} \cdot \nabla \mathrm{u}\right)=-\nabla \mathrm{p}+\mu \nabla^{2} \mathrm{u}+\mathrm{f} \\
& -\nabla \cdot \mathrm{u}=0
\end{aligned}
$$

We assumed that blood can be described as incompressible Newtonian fluid with a density of $1046 \mathrm{~kg} / \mathrm{m}^{3}$ and a viscosity of 0.04 dynes $/ \mathrm{cm}^{2}$. The wall of the blood vessels supposed to be rigid without slipping.

\section{Noninvasive CAFA measurement}

Fractional flow reserve was an important indicator for ischemia of the carotid artery. Carotid arterial functional assessment (CAFA) index was derived from the concept used in coronary arteries and was defined as the ratio of flow in the stenotic vessel to the flow in the same vessel without stenosis.

$$
\operatorname{CAFA}_{v}=\frac{\mathrm{Q}_{\mathrm{H}}^{\mathrm{S}}}{\mathrm{Q}_{\mathrm{H}}^{\mathrm{N}}}
$$

where $\mathrm{Q}_{\mathrm{H}}^{\mathrm{S}}$ is the flow rate at the stenotic vessel, $\mathrm{Q}_{\mathrm{H}}^{\mathrm{N}}$ is the flow rate at the same normal vessel. When applied to the suspected stenotic arteries, the normal artery model can be 
determined as the arteries with stenosis removed. According to the theory proposed by Pijls et al. the CAFA may have an approximate simplified expression based on directly measured data [11].

$$
\operatorname{CAFA}_{p}=\frac{\mathrm{mP}_{\mathrm{d}}}{\mathrm{mP}_{\mathrm{a}}}
$$

\section{Statistical methods}

Eighteen points were selected through the CFD simulation and pressure wire derived pull-back curves respectively, and the $\mathrm{Pd} / \mathrm{Pa}$ was calculated one to one. The paired t-test, Pearson correlation and Bland-Altman plots were used to assess the accordance between the CFD simulation and invasive measurements. A P-value less than 0.05 was deemed statistically significant. All the analyses were performed on the SPSS (version 14, Chicago, IL, USA) and MedCalc Software (MedCalc, Mariakerke, Belgium).

\section{Results}

The perfusion data of left and right ICA territories measured by the PCASL was presented at Table 1 and Fig. 1. Compared with the contralateral hemisphere, CBF with 1.0 PLD decreased slightly in the involved left ICA territories, especially in the left frontoparietal. With the increasing of PLD, CBF increased in the involved territories and the final $\mathrm{CBF}$ was close to the right hemisphere in spite of a slightly prolonged ATT in left.

As showed in Fig. 2a, the pressure ratio $(\mathrm{Pd} / \mathrm{Pa})$ calculated from DSA data set was presented, as well as the ones measured by pressure wire; and two curves both appeared a sharpen decrease around $20 \mathrm{~mm}$, where the stenosis is. Finally, the invasive CAFA measured by guide-wire was 0.937 , and the noninvasive CAFA was calculated as 0.942 within $14 \mathrm{~s}$ using CFD simulation.

Pressure profile obtained by invasive way was presented as comparison of the simulation. As showed in Fig. 2b, the pressure curves calculated by simulation and measured by pressure guide-wire were presented respectively, and were overlapped well. Furthermore, the simulation results and the pressure-wired measurements showed good agreement $(r=0.839, P=0.001$, Fig. 3a), and no significant difference was observed between two methods $(\mathrm{P}=0.09)$. Additionally, the Bland-Altman plot presented a slight systematic overestimation of calculated $\mathrm{Pd} / \mathrm{Pa}$ based DSA (mean difference -0.007 , standard deviation 0.017, Fig. 3b).

Table 1 The results of CBF ( $\mathrm{ml} / 100 \mathrm{~g} / \mathrm{min}$ ) and ATT (s) in ICA territories

\begin{tabular}{|c|c|c|c|c|c|c|}
\hline & \multicolumn{3}{|c|}{ Fronto-temporal } & \multicolumn{3}{|l|}{ Fronto-parietal } \\
\hline & Left (involved) & Right (normal) & $\Delta \%$ & Left (involved) & Right (normal) & $\Delta \%$ \\
\hline $\mathrm{CBF}(\mathrm{PLD}=1.0 \mathrm{~s})$, & 51.76 & 52.93 & -2.21 & 32.54 & 36.98 & -12.0 \\
\hline $\mathrm{CBF}(\mathrm{PLD}=2.0 \mathrm{~s})$ & 50.61 & 49.73 & 1.77 & 49.55 & 49.78 & -0.46 \\
\hline $\mathrm{CBF}(\mathrm{PLD}=3.0 \mathrm{~s})$ & 52.38 & 48.02 & 9.08 & 54.76 & 53.05 & 3.22 \\
\hline Final CBF & 51.58 & 50.23 & 2.69 & 45.62 & 46.6 & 2.10 \\
\hline ATT & 1.23 & 1.13 & 8.85 & 1.40 & 1.31 & 6.87 \\
\hline
\end{tabular}

CBF cerebral blood flow, PLD post-labeling delay, ATT arterial transit time, ICA internal carotid artery

$\triangle \%$ was left-right percentage difference 


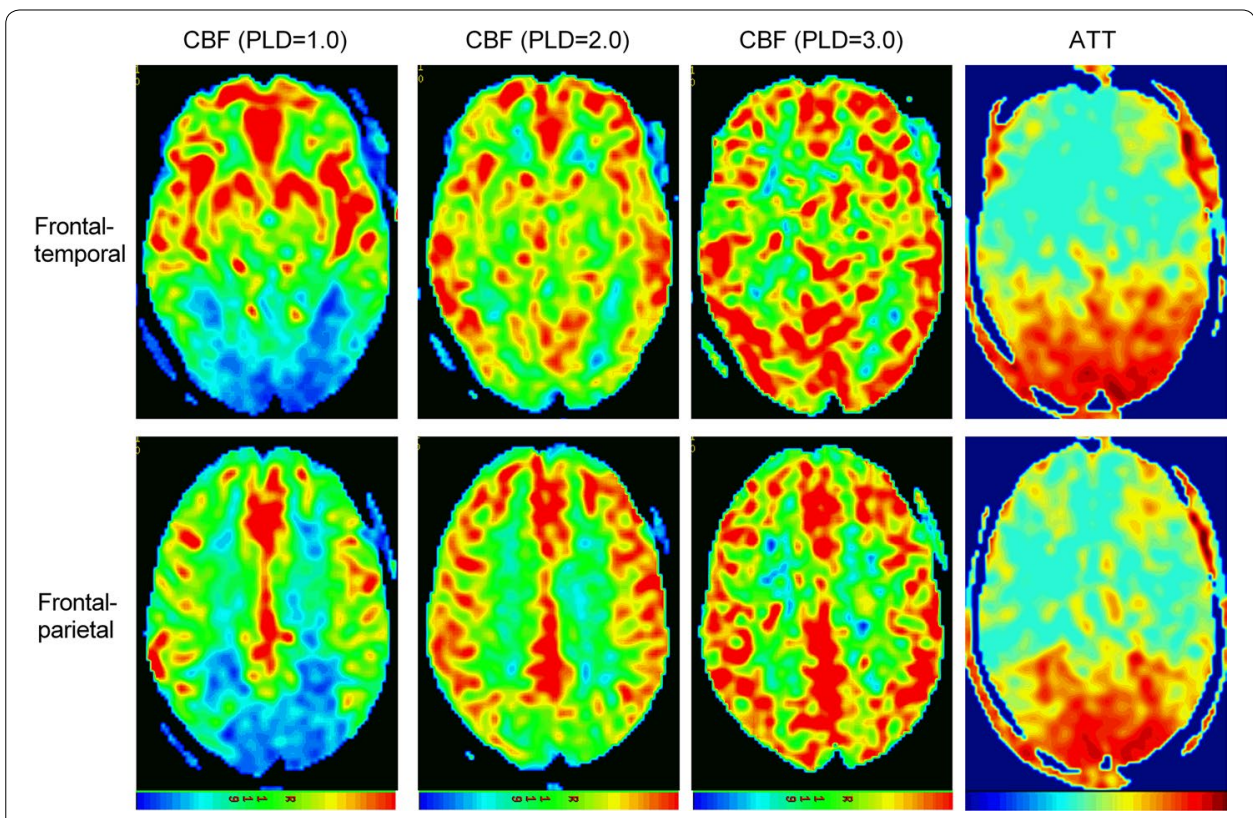

Fig. 1 The parametric CBF and ATT maps of presented case. CBF with 1.0 PLD decreased slightly in the involved left ICA territories compared with the contralateral hemisphere, especially in the left fronto-parietal. With the increasing of PLD, CBF increased in the involved territories and was close to the right hemisphere in spite of a slightly prolonged ATT in left
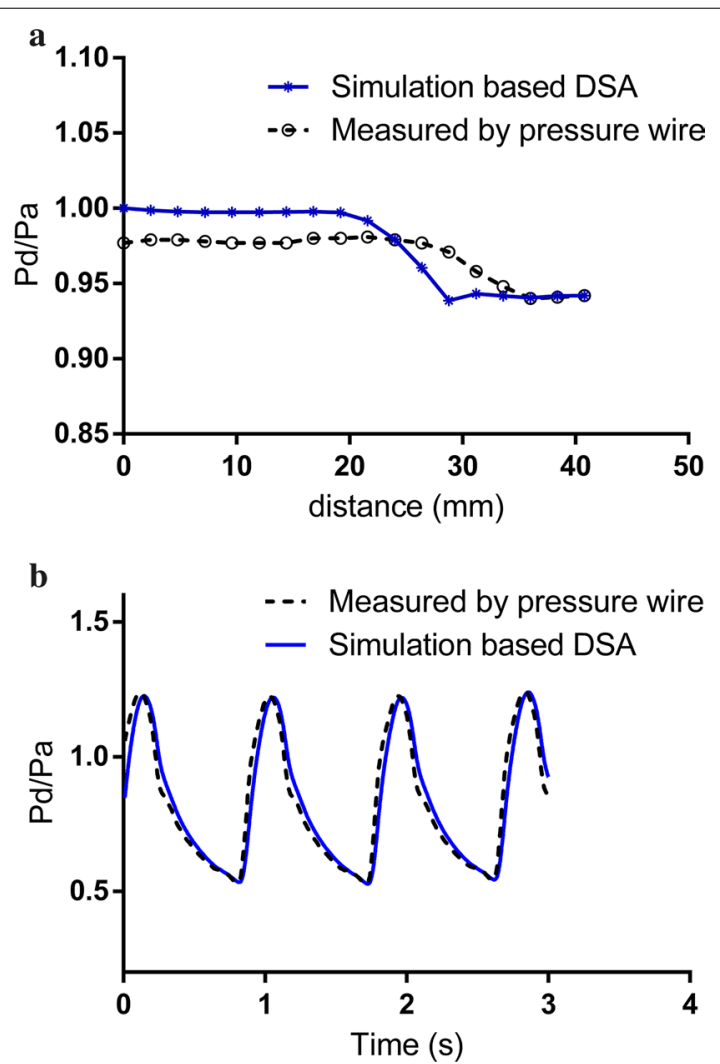

Fig. 2 Pressure profiles calculated with CFD simulation and measured by pressure wire. a Pressure ratio versus distance along the centerline. $\mathbf{b}$ Pressure ratio versus time at the boundary 

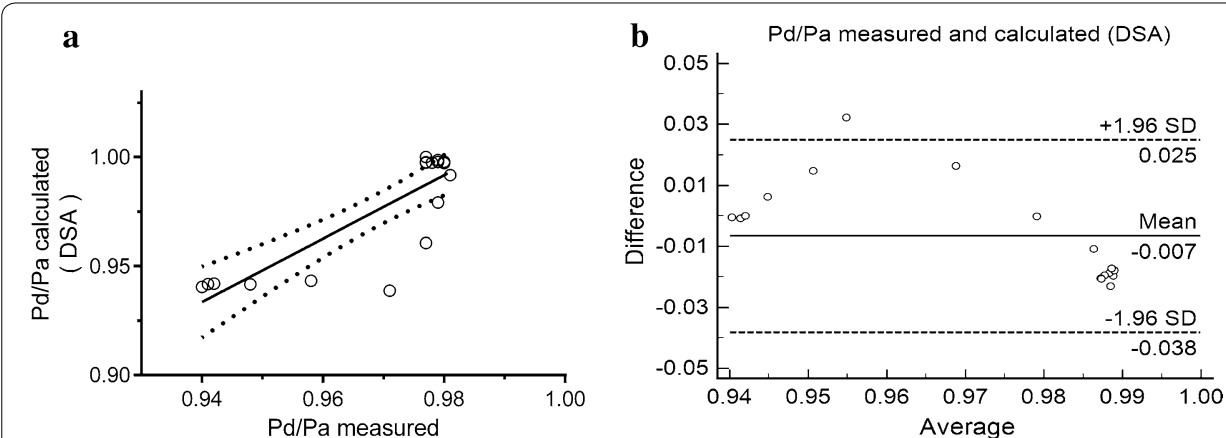

Fig. 3 Comparison of pressure gradient ratio ( $\mathrm{Pd} / \mathrm{Pa}$ ) between CFD simulation and invasive measurement. Pearson correlation analysis $\mathbf{a}$ and Bland-Altman plots $\mathbf{b}$ of calculated $\mathrm{Pd} / \mathrm{Pa}$ based DSA data sets compared with the measured result; $r$ was 0.839 with significant difference $(P=0.001)$, and mean difference was -0.007 with standard deviation 0.017

Furthermore, we also depicted a set of hemodynamics distributions of the carotid artery with three typical angles $\left(0^{\circ}, 60^{\circ}, 120^{\circ}\right)$ in Fig. 4 . The hemodynamic distributions varied with the decrease of lumen radius. At the site of stenosis, the pressure decreased nearly $400 \mathrm{~Pa}$ while the velocity increased nearly $0.5 \mathrm{~m} / \mathrm{s}$; as for the wall shear stress (WSS), it increased nearly $30 \mathrm{~Pa}$.

\section{Discussion}

The treatment for patients with asymptomatic carotid artery stenosis is highly controversial in clinic because of lacking the direct and quantitative indicators to assess the risk of stroke in brain [3, 31,32]. Hemodynamic analysis plays an important role in making decision before the artery revascularization $[9,10]$. In this case, we performed pCASL MRI with multiple PLD times to evaluate the hemodynamic characteristics in brain together with an invasive pressure gradient measurement of carotid stenosis and an introduced CFD method based on the DSA data.

Our results showed that CBF of the involved left ICA territories at 1.0 PLD decreased compared with the contralateral hemisphere, especially in the left fronto-parietal. However, with the increasing of PLD, CBF increased in the involved left ICA territories, and the final CBF was close to the right hemisphere in spite of a slightly prolonged ATT in left. The compromised cerebral blood flow (CBF) caused by internal carotid stenosis indicates a risk for future ischemic stroke in brain [9, 24]. Most ASL studies employ a single post-labeling delay (PLD) time between 1.5 and $2.0 \mathrm{~s}$ to compute the CBF [26]. However, ASL is highly susceptible to the arterial transit time (ATT) [28]. When a prolonged ATT caused by arterial stenosis and collateral blood flow is greater than the employed PLD, the CBF will be underestimated, and it would be ideal to apply ASL with multiple PLDs to estimate the CBF and ATT simultaneously [29]. In previous perfusion studies on carotid stenosis, a "misery perfusion" was defined as CBF $<35 \mathrm{ml} / 100 \mathrm{~g}$ / min or ATT> $7.89 \mathrm{~s}$, and normal left-right difference were known to vary $\pm 10 \%[33$, 34]. Finally, based our results of ASL, we did not think this patient has the high risk of ischemic stroke even though the degree of narrowing is severe; and the absence of intraplaque hemorrhage and lipid-rich necrotic core reduce the risk of rupture. Finally, we 


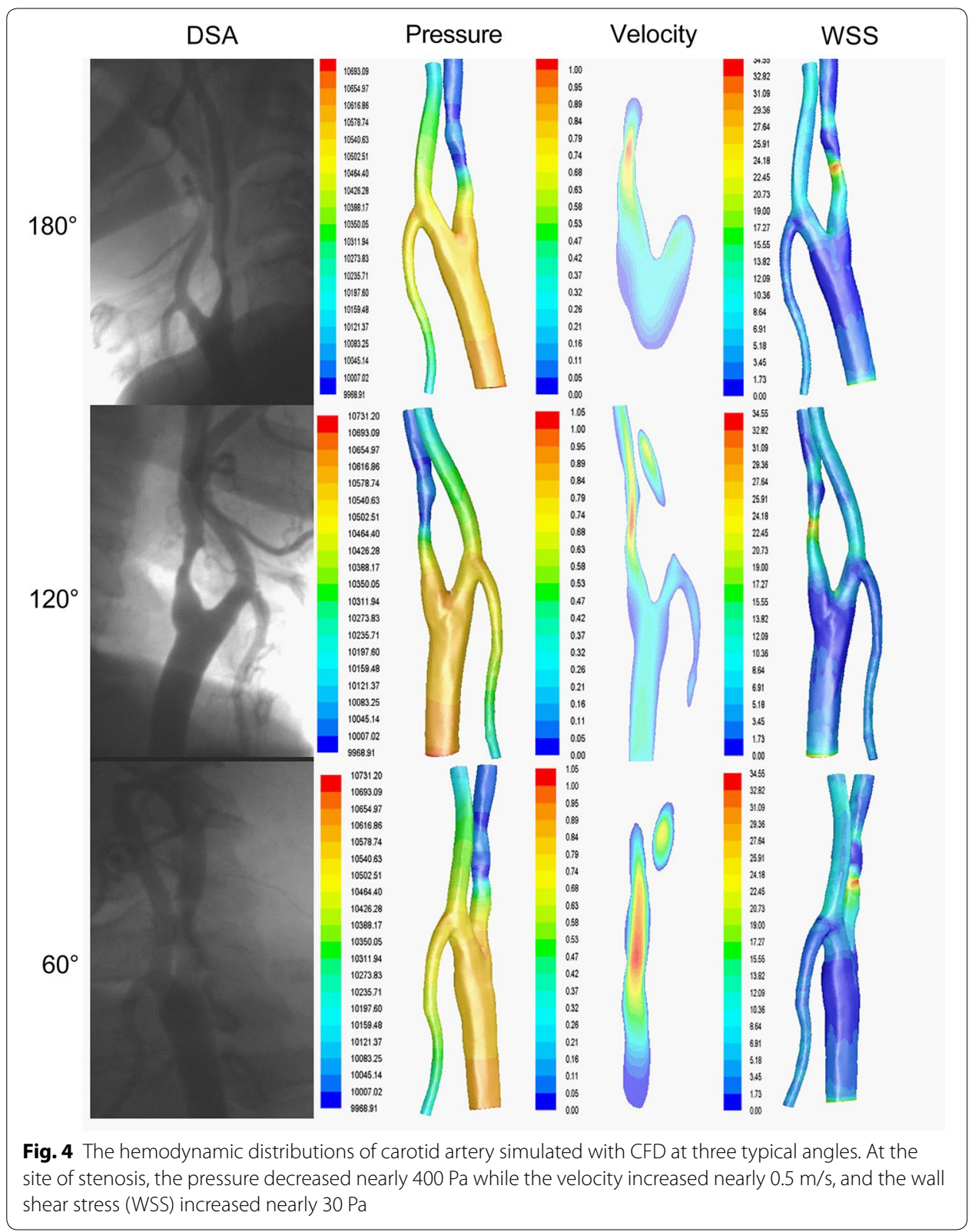

excluded the choice of carotid stenting or endarterectomy, and treated him with intensive medical therapy adding aspirin, clopidogrel, diuretic, and statin.

Invasive functional assessment based on pressure gradient has been proved to be applicable in internal carotid artery quantitatively [12]. In the presented case, even though the left carotid stenosis was severe (estimated about 70\%), the acquired invasive CAFA was 0.973 . According to Han's research, revascularization was performed in indicated lesion only if the pressure gradient ratio was 0.7 or less which had been widely used in coronary stenosis [12]. While Han also suggested that the measured $\mathrm{mPd} / \mathrm{mPa}$ in carotid artery is not equal to the FFR in coronary due to the lack of induced hyperemia [12]. But Liu argued that the resistance of cerebral and neck vascular is low and constant 
which should be neglected, and the induced hyperemia is not necessary [13]. However, pressure-based carotid functional assessment could not be widely used to indicate the management in the clinic, because it lacks enough evidence to identify the diagnostic criterion. Multi-delay multi-parametric pCASL may be an effective way to provide a direct and quantitative evidence to identify the diagnostic standard of CAFA.

The high cost of pressure wire and the invasive medical operation may hinder the application of hemodynamic measurement in clinic [14]. As an alternative method, we introduced a CFD simulations based on DSA data in this study, and calculated the CAFA to help diagnose the ischemia-related carotid artery stenosis. Our results showed a good accuracy between pressure simulation and pressure-wired measurement. Furthermore, using the velocity derived from DSA data to simplify the computational model, we obtained the reliable results within $15 \mathrm{~s}$ which made it prepared for the fast analysis in clinic. Previous studies of CFD simulation have focused on coronary artery to assess the hemodynamic characteristics, and fast computation of pressure ratio from coronary angiography, acquired with or without pharmacological hyperemia-induction, is feasible $[17,35]$. In our study, the calculated CAFA is 0.942 based DSA data. According to Liu's study on the functional assessment of stenotic carotid artery by CFD-based pressure gradient evaluation, the value of 0.88 was suggested as an indicator to classify the severe and mild-to-moderate stenosis [21]. However, in Liu's research, the severity of carotid stenosis was categorized based on peak systolic velocity (PSV) and morphology measured by ultrasound, and it neglected the compensation of collateral circulation and lack direct evidence to identify the ischemia-related carotid stenosis quantitatively. Therefore, with its advantage in saving costs, reducing procedure time and lowering invasive risk, it is necessary to investigate the potential of CFD-based CAFA in screening the ischemia-related carotid artery stenosis, and use the advanced multi-delay multiparametric PCASL-MRI as a reference.

Even though the selected points showed good agreement between simulation results and the pressure-wired measurements, there was a constant difference between the CFD and in vivo results in Fig. 2a, as well as an outlier in Fig. 3b. As the pressure recording is only related to the time and speed when the pressure sensor was pulled back, and the pull-back curves was estimated according to the recording data, it is difficult to realize the absolutely precise fitting during the data processing. Besides, these phenomena may also be caused by the limited resolution of the carotid DSA, especially the region proximal to the stenosis, which may lead to the deviation between reconstructed geometries and in vivo anatomy. Furthermore, only the cross-sectional area was used to calculate the average flow of internal carotid artery, the volume of the simulation domain was not taken into the consideration which may also cause the error. Further adjustment taking a correction of the actual vascular volume into the simulation domain is required, and the improvement of the image quality is also necessary to reduce the deviation caused by geometric effects.

As a preliminary study, we restricted to only one typical patient's imaging data sets to verify the method of CFD simulation in carotid stenosis. Actually more cases were necessary for a full validation, and a single center study including normal and ischemia cases is needed in the future. Besides, the measurement of CAFA we implemented was 
according to Han's introduced procedure [12], and further research on the establishment of standard protocol is necessary.

\title{
Conclusions
}

Despite these limits, our study introduced a noninvasive method to evaluate the hemodynamic disturbance of asymptomatic carotid artery stenosis quantitatively. Based on the DSA data, we simplified the CFD simulation and calculated the pressure-based CAFA index efficiently. The calculated pressure gradient ratio showed a good consistence with the pressure-wired measurement, and the noninvasive CAFA index revealed a functionally nonsignificant stenosis in a 65-year-old man with severe asymptomatic unilateral ICA stenosis, which can be verified by cerebral multi-delay multi-parametric ASL-MRI. Therefore, the DSA based simulation has the potential to be used in studying the relationship between hemodynamic disorder in ICA stenosis and subsequent perfusion variations instead of costly pressure wire. Our current research provided a possibility of noninvasive pressure-based CAFA in screening asymptomatic ischemia-causing carotid stenosis. Further research including normal and ischemia cases should been put on the agenda.

\begin{abstract}
Abbreviations
ICA: internal carotid artery; DSA: digital subtracted angiography; CAFA: carotid arterial functional assessment; CFD: computational fluid dynamic; pCASL: pseudo-continuous arterial spin labeling; CBF: cerebral blood flow; ATT: arterial transit time; CTA: computed tomography angiography; FFR: fraction flow reserve; CE-MRA: contrast-enhancement MR angiography; PLD: post-labeling delay; T2DM: type 2 diabetes mellitus; WSS: wall shear stress.
\end{abstract}

\section{Authors' contributions}

DZ, CZS and HYQ contributed to the data collecting; DZ, PCX and XL contributed to the methodology; DZ and PCX contributed to the analysis of results; DZ and PCX contributed to write the original draft of the manuscript; CZS and HYZ contributed to review and editing of the manuscript; CZS, HYZ, LPL and WHH contributed to Supervision; LPL, CZS and HYZ contributed to Project Administration; LPL, HYZ contributed to Funding Acquisition. All authors read and approved the final manuscript.

\section{Author details}

${ }^{1}$ Department of Medical Imaging Center, The First Affiliated Hospital, Jinan University, No. 613, Huangpu Road West, Tianhe District, Guangzhou 510630, Guangdong Province, China. ${ }^{2}$ Department of Anatomy, Guangdong Provincial Key Laboratory of Medical Biomechanics, School of Basic Medical Science, Southern Medical University, Guangzhou 510515, China. ${ }^{3}$ Institute of Advanced Computing and Digital Engineering, Shenzhen Institutes of Advanced Technology, 1068 Xueyuan Ave, Xili University Town, Nanshan, Shenzhen 518055, Guangdong Province, China.

\section{Acknowledgements}

Not applicable.

\section{Competing interests}

The authors declare that they have no competing interests.

Availability of data and materials

The datasets analyzed during the current study are not publicly available because of the further analysis of the datasets in our research but are available from the corresponding author upon reasonable request.

\section{Ethics approval and consent to participate}

The study was approved by the ethical review committee of First Affiliated Hospital of Jinan University (Guangzhou, Guangdong, China) (Study Number: 2016025) and was conducted in conformance with the Helsinki Declaration. Since this study is a retrospective study, the informed consent was waived and anonymized data was used for analysis.

\section{Funding}

This study is funded by Collaborative Innovation Program of Hong Kong and Guangdong Province, the Guangdong Science and Technique Department (2016A050503032, 2014A020212689), National Key Research and Development Program of China (2016YFC1301700), and the Science Technology and Innovation Committee of Shenzhen for Research Projects (JCYJ20151030151431727).

\section{Publisher's Note}

Springer Nature remains neutral with regard to jurisdictional claims in published maps and institutional affiliations. 
Received: 13 December 2017 Accepted: 7 March 2018

Published online: 12 March 2018

\section{References}

1. Yang F, Liu L, Li M, Li M, Yin Q, Guo R, et al. Pattern of cerebrovascular atherosclerotic stenosis in older Chinese patients with stroke. J Clin Neurosci. 2013;20(7):979-83.

2. de Weerd M, Greving JP, de Jong AW, Buskens E, Bots ML. Prevalence of asymptomatic carotid artery stenosis according to age and sex: systematic review and metaregression analysis. Stroke. 2009;40(4):1105-13.

3. Spence JD, Pelz D, Veith FJ. Asymptomatic carotid stenosis: identifying patients at high enough risk to warrant endarterectomy or stenting. Stroke. 2014;45(3):655-7.

4. Calvet D, Amar L, Rossi GP, Laurent S, Dominiczak AF, Turc G, et al. Case of asymptomatic carotid artery stenosis in a hypertensive patient. Hypertension. 2017;69(6):985-91.

5. Heck DV, Roubin GS, Rosenfield KG, Gray WA, White CJ, Jovin TG, et al. Asymptomatic carotid stenosis: medicine alone or combined with carotid revascularization. Neurology. 2017;88(21):2061-5.

6. Kasner SE, Chimowitz MI, Lynn MJ, Howlett-Smith H, Stern BJ, Hertzberg VS, et al. Predictors of ischemic stroke in the territory of a symptomatic intracranial arterial stenosis. Circulation. 2006;113(4):555-63.

7. Silvennoinen HM, Ikonen S, Soinne L, Railo M, Valanne L. CT angiographic analysis of carotid artery stenosis: comparison of manual assessment, semiautomatic vessel analysis, and digital subtraction angiography. AJNR Am J Neuroradiol. 2007:28(1):97-103.

8. Netuka D, Belsan T, Broulikova K, Mandys V, Charvat F, Malik J, et al. Detection of carotid artery stenosis using histological specimens: a comparison of CT angiography, magnetic resonance angiography, digital subtraction angiography and Doppler ultrasonography. Acta Neurochir (Wien). 2016;158(8):1505-14.

9. Silvestrini M, Vernieri F, Pasqualetti P, Matteis M, Passarelli F, Troisi E, et al. Impaired cerebral vasoreactivity and risk of stroke in patients with asymptomatic carotid artery stenosis. JAMA. 2000;283(16):2122-7.

10. Seo HJ, Pagsisihan JR, Paeng JC, Choi SH, Cheon GJ, Chung JK, et al. Hemodynamic significance of internal carotid or middle cerebral artery stenosis detected on magnetic resonance angiography. Yonsei Med J. 2015;56(6):1686-93.

11. Pijls NH, De Bruyne B, Peels K, Van Der Voort PH, Bonnier HJ, Bartunek JKJJ, et al. Measurement of fractional flow reserve to assess the functional severity of coronary-artery stenoses. N Engl J Med. 1996;334(26):1703-8.

12. Han YF, Liu WH, Chen XL, Xiong YY, Yin Q, Xu GL, et al. Severity assessment of intracranial large artery stenosis by pressure gradient measurements: a feasibility study. Catheter Cardiovas Interv. 2016;88(2):255-61.

13. Liu CP, Ling YH, Kao HL. Use of a pressure-sensing wire to detect sequential pressure gradients for ipsilateral vertebral and subclavian artery stenoses. AJNR Am J Neuroradiol. 2005;26(7):1810-2.

14. Siebert U, Arvandi M, Gothe RM, Bornschein B, Eccleston D, Walters DL, et al. Improving the quality of percutaneous revascularisation in patients with multivessel disease in Australia: cost-effectiveness, public health implications, and budget impact of FFR-guided PCI. Heart Lung Circ. 2014;23(6):527-33.

15. Trivedi S, Aroney CN, Walters DL, Bett JH. Measurement of myocardial fractional flow reserve is a cost-effective way to identify coronary artery lesions of indeterminate severity that warrant revascularisation. Heart Lung Circ. 2005; 14(4):239-41.

16. Koo BK, Erglis A, Doh JH, Daniels DV, Jegere S, Kim HS, et al. Diagnosis of ischemia-causing coronary stenoses by noninvasive fractional flow reserve computed from coronary computed tomographic angiograms. Results from the prospective multicenter DISCOVER-FLOW (Diagnosis of ischemia-causing stenoses obtained via noninvasive fractional flow reserve) study. J Am Coll Cardiol. 2011;58(19):1989-97.

17. Shi C, Zhang D, Cao K, Zhang T, Luo L, Liu X, et al. A study of noninvasive fractional flow reserve derived from a simplified method based on coronary computed tomography angiography in suspected coronary artery disease. Biomed Eng Online. 2017;16(1):43.

18. Liu X, Gao Z, Xiong H, Ghista D, Ren L, Zhang H, et al. Three-dimensional hemodynamics analysis of the circle of Willis in the patient-specific nonintegral arterial structures. Biomech Model Mechanobiol. 2016;15(6):1439-56.

19. Xue YJ, Gao PY, Duan Q, Lin Y, Dai CB. Preliminary study of hemodynamic distribution in patient-specific stenotic carotid bifurcation by image-based computational fluid dynamics. Acta Radiol. 2008;49(5):558-65.

20. Shih TC, Lin YH, Ho YJ, Hsiao HD, Huang YH, Huang TC. Hemodynamic analysis of vascular stenting treatment outcome: computational fluid dynamics method vs optical flow method. J Xray Sci Technol. 2012;20(4):469-81.

21. Liu X, Zhang H, Ren L, Xiong H, Gao Z, Xu P, et al. Functional assessment of the stenotic carotid artery by CFD-based pressure gradient evaluation. Am J Physiol Heart Circ Physiol. 2016;311(3):H645-53.

22. Bokkers RP, van der Worp HB, Mali WP, Hendrikse J. Noninvasive MR imaging of cerebral perfusion in patients with a carotid artery stenosis. Neurology. 2009;73(11):869-75.

23. Pfefferbaum A, Chanraud S, Pitel AL, Shankaranarayanan A, Alsop DC, Rohlfing T, et al. Volumetric cerebral perfusion imaging in healthy adults: regional distribution, laterality, and repeatability of pulsed continuous arterial spin labeling (PCASL). Psychiatry Res. 2010;182(3):266-73.

24. Hosoda K. The Significance of cerebral hemodynamics imaging in carotid endarterectomy: a brief review. Neurol Med Chir. 2015;55(10):782-8.

25. Zaharchuk G. Arterial spin-labeled perfusion imaging in acute ischemic stroke. Stroke. 2014:45(4):1202-7.

26. Grade M, Hernandez Tamames JA, Pizzini FB, Achten E, Golay X, Smits M. A neuroradiologist's guide to arterial spin labeling MRI in clinical practice. Neuroradiology. 2015;57(12):1181-202.

27. Wang DJ, Alger JR, Qiao JX, Gunther M, Pope WB, Saver JL, et al. Multi-delay multi-parametric arterial spin-labeled perfusion MRI in acute ischemic stroke-comparison with dynamic susceptibility contrast enhanced perfusion imaging. Neurolmage Clin. 2013;3:1-7. 
28. Alsop DC, Detre JA, Golay X, Gunther M, Hendrikse J, Hernandez-Garcia L, et al. Recommended implementation of arterial spin-labeled perfusion MRI for clinical applications: a consensus of the ISMRM perfusion study group and the European consortium for ASL in dementia. Magn Reson Med. 2015;73(1):102-16.

29. Maclntosh BJ, Lindsay AC, Kylintireas I, Kuker W, Gunther M, Robson MD, et al. Multiple inflow pulsed arterial spinlabeling reveals delays in the arterial arrival time in minor stroke and transient ischemic attack. AJNR Am J Neuroradiol. 2010;31(10):1892-4.

30. Wang R, Yu S, Alger JR, Zuo Z, Chen J, Wang R, et al. Multi-delay arterial spin labeling perfusion MRI in moyamoya disease-comparison with CT perfusion imaging. Eur Radiol. 2014;24(5):1135-44.

31. Marquardt L, Geraghty OC, Mehta Z, Rothwell PM. Low risk of ipsilateral stroke in patients with asymptomatic carotid stenosis on best medical treatment: a prospective, population-based study. Stroke. 2010;41(1):e11-7.

32. Starke RM. Optimal management of patients with asymptomatic carotid stenosis. Neurology. 2017:88(21):1988-9.

33. Yamauchi H, Higashi T, Kagawa S, Kishibe Y, Takahashi M. Chronic hemodynamic compromise and cerebral ischemic events in asymptomatic or remote symptomatic large-artery intracranial occlusive disease. AJNR Am J Neuroradiol. 2013;34(9):1704-10

34. Waaijer A, van der Schaaf IC, Velthuis BK, Quist M, van Osch MJP, Vonken EPA, et al. Reproducibility of quantitative CT brain perfusion measurements in patients with symptomatic unilateral carotid artery stenosis. Am J Neuroradiol. 2007;28(5):927-32.

35. Yoon YE, Choi JH, Kim JH, Park KW, Doh JH, Kim YJ, et al. Noninvasive diagnosis of ischemia-causing coronary stenosis using CT angiography: diagnostic value of transluminal attenuation gradient and fractional flow reserve computed from coronary CT angiography compared to invasively measured fractional flow reserve. JACC Cardiovasc Imag. 2012;5(11):1088-96.

Submit your next manuscript to BioMed Central and we will help you at every step:

- We accept pre-submission inquiries

- Our selector tool helps you to find the most relevant journal

- We provide round the clock customer support

- Convenient online submission

- Thorough peer review

- Inclusion in PubMed and all major indexing services

- Maximum visibility for your research

Submit your manuscript at www.biomedcentral.com/submit 